\title{
Análise dos Motivos dos Jovens e Adultos consumirem Álcool
}

\author{
Fernanda de Jesus Soares ${ }^{1}$; Daiana Cirqueira Oliveira ${ }^{2}$; Paula Rocha Oliveira ${ }^{3}$; Tatiane Souza Lima ${ }^{4}$; \\ Adriana Lopes Rodrigues Alves ${ }^{5}$; Matheus Lemos Silva ${ }^{6}$ Stênio Fernando Pimentel Duarte
}

\begin{abstract}
Resumo: O álcool é uma droga lícita responsável por deprimir o Sistema Nervoso Central e é tida como uma das substâncias com maior grau de consumo a nível mundial, a Organização Mundial da Saúde (WHO) traz que em média dois bilhões de pessoas fazem o consumo de bebidas alcoólicas, compreendendo tanto o uso por dependentes quanto o uso social. A indústria do marketing do álcool nos dias atuais age globalmente, tanto em países industrializados quando nos que ainda estão em desenvolvimento. Entretanto existem fatores além do marketing e o fácil acesso ao álcool, que podem levar ao seu consumo, como o incentivo através de amizades, isso se deve principalmente a necessidade de se enquadrar em grupos na sociedade. Esse artigo traz como objetivo a análise desses motivos pelos quais os jovens e adultos consomem o álcool. Metodologia: O presente estudo faz parte do projeto "Identificando o perfil epidemiológico da Obesidade em Vitória da Conquista" idealizado pelo Núcleo de Extensão, Pesquisa e Estudo de Doenças Crônicas - NEPEDC da Faculdade Independente do Nordeste. O procedimento portou-se inicialmente através da aplicação de questionário, instrumento para coleta de dados que continha perguntas pertinente ao estudo, seguido de uma segunda entrevista realizada via ligação telefônica. Esses procedimentos foram realizados com indivíduos aleatórios, com faixa etária entre 20 a 59 anos, considerados jovens adultos. Resultados: A prevalência entre jovens e adultos é de $16 \%$ Jovens adultos, 59\% Adultos e $21 \%$ Adultos velhos. A idade de inicio do consumo de álcool, 61\% relataram que foi ainda na adolescência e 39\% já na fase adulta. Em relação aos motivos que levam os jovens e adultos consumirem álcool 57\% disseram ser para interagir com colegas, $16 \%$ para distrair-se de alguma situação/ problema, $14 \%$ para aliviar tensão/stress, $7 \%$ disseram beber por lazer, $4 \%$ em expectativa em algo positivo e $2 \%$ para aumento de confiança em situações com outras pessoas. Em relação aos meios que induziram os pesquisados a consumirem bebidas alcoólicas pela primeira vez, $68 \%$ disseram ter experimentado através de amigos, $23 \%$ por interesse próprio/curiosidade e $9 \%$ por meio de parentes. Nos resultados, dos lugares em que os pesquisados consomem álcool com mais frequência, $34 \%$ disseram ser em casa, 31\% em bares, $29 \%$ em casas de amigos e $6 \%$ em clubes. Conclusão: Esta pesquisa foi voltada para análise dos motivos que levam os jovens e adultos a consumirem álcool, e em concordância com a avaliação dos dados e as informações literárias, é possível concluir que este consumo possa ser influenciado por diversos fatores. Notasse que a sociedade impõe de certo modo, normas e valores que seriam essenciais para uma harmonia social e hoje o uso de bebidas alcoólicas na sociedade está diretamente ligado a própria vivência que assegura uma vida social.
\end{abstract}

Descritores: Consumo de álcool. Influência de amizades. Motivos que levam ao consumo.

\section{Analysis of Youth and Adult Motivation Consume Alcohol}

\begin{abstract}
Alcohol is a licit drug responsible for depressing the Central Nervous System and is considered one of the substances with the highest consumption level worldwide, the World Health Organization (WHO) brings that on average two billion people drink Alcoholics, including both dependents and social use. The alcohol marketing industry nowadays acts globally in both industrialized and developing countries. However there are factors besides marketing and easy access to alcohol, which can lead to their consumption, such as the incentive through friendships, this is mainly due to the need to fit into groups in society. This article aims to analyze the reasons why young people and adults consume alcohol. Methodology: This study is part of the project "Identifying the
\end{abstract}

\footnotetext{
${ }^{1}$ Graduanda do Curso de Farmácia da Faculdade Independente do Nordeste (FAINOR). E-mail: feeh_23@hotmail.com

${ }^{2}$ Graduanda do Curso de Farmácia da Faculdade Independente do Nordeste (FAINOR). E-mail: daicirqueira@ hotmail.com

${ }^{3}$ Graduanda do Curso de Farmácia da Faculdade Independente do Nordeste (FAINOR). E-mail: paulafarma23@gmail.com

${ }^{4}$ Graduanda do Curso de Farmácia da Faculdade Independente do Nordeste (FAINOR). E-mail: tatilisouza21@ gmail.com

${ }^{5}$ Licenciatura em História, mestrado profissional em Gestão de Organizações Aprendentes, pela Universidade Federal da Bahia, atualmente é professora de Ética, Sociologia e História da Moda no ensino superior. E-mail: adrylopes2@ gmail.com

${ }^{6}$ Graduando do curso de Nutrição da Faculdade de Tecnologias e Ciências, com atuação nas áreas de Saúde Coletiva e Ciências dos alimentos. E-mail: Matheus.nutri3@gmail.com

${ }^{7}$ Graduado em Ciências Biológicas pela Universidade do Estado do Rio de Janeiro, mestrado em Ciências e doutorado em Ciências ( Fisiopatologia Clínica e Experimental) pela Universidade do Estado do Rio de Janeiro, atualmente é docente do curso de Farmácia na Faculdade Independente do Nordeste - FAINOR, Vitória da Conquista/BA. E-mail: steniofernando@ gmail.com
} 
Epidemiological Profile of Obesity in Vitória da Conquista" idealized by the Nucleus of Extension, Research and Study of Chronic Diseases - NEPEDC, Faculdade Independente do Nordeste. The procedure was carried out initially through the application of a questionnaire, an instrument for data collection that contained questions pertinent to the study, followed by a second interview conducted via phone call. These procedures were performed with random individuals, with ages ranging from 20 to 59 years, considered young adults. Results: The prevalence among youth and adults is $16 \%$ Young adults, 59\% Adults and 21\% Old adults. The age at onset of alcohol consumption, $61 \%$ reported that it was still in adolescence and $39 \%$ already in adulthood. $57 \%$ said they were to interact with colleagues, $16 \%$ to distract themselves from some situation / problem, $14 \%$ to relieve tension / stress, $7 \%$ said to drink for leisure, $4 \%$ In expectation of something positive and $2 \%$ to increase confidence in situations with other people. Regarding the means that induced the respondents to consume alcoholic beverages for the first time, $68 \%$ said they had experimented through friends, $23 \%$ self-interest / curiosity and 9\% through relatives. In the results, of the places where respondents consume alcohol more frequently, 34\% said they were at home, 31\% in bars, $29 \%$ in friends' houses and $6 \%$ in clubs. Conclusion: This research was aimed at analyzing the reasons that lead adolescents and adults to consume alcohol, and in agreement with the evaluation of data and literary information, it is possible to conclude that this consumption can be influenced by several factors. Note that society imposes in a certain way, norms and values that would be essential for a social harmony and today the use of alcoholic beverages in society is directly linked to the very experience that ensures a social life.

Keywords: Alcohol consumption. Influence of friendships. Reasons that lead to consumption.

\section{Introdução}

O álcool é uma droga lícita responsável por deprimir o Sistema Nervoso Central e é tida como uma das substâncias com maior grau de consumo a nível mundial, a Organização Mundial da Saúde (WHO) traz que em média dois bilhões de pessoas fazem o consumo de bebidas alcoólicas, compreendendo tanto o uso por dependentes quanto o uso social (G. A. REIS, GÓIS, ALVES, \& PARTATA, 2014)(A. Reis, Barros, Fonseca, \& Parreira, 2011).

Sabe-se que o álcool é uma das poucas drogas psicotrópicas que possui admissão em seu consumo, sendo até incentivada pela sociedade brasileira, logo que o álcool é socialmente encarado de forma diferenciada quando correlacionado com outras drogas, tendo o seu consumo como uma condição comum no cotidiano, este engloba cerca de 10 a $12 \%$ da população brasileira adulta (VENTUROSO, 2015).

Sua ingestão ocorre desde a antiguidade, na época dos gregos, onde essa prática era considerada hábitos de sociabilidade, eles bebiam o vinho no anoitecer sucessivamente às refeições, sendo considerado um ato de excentricidade. E com o passar dos anos essa praxe passou a ser naturalizada e até incentivada na maioria das crenças e culturas (Faria, Vendrame, SILVA, \& PINSKY, 2011). 
A indústria do marketing do álcool nos dias atuais age globalmente, tanto em países industrializados quando nos que ainda estão em desenvolvimento (ACSERALD et al., 2012). O mercado associa o consumo das bebidas com álcool a serem vendidas com a cultura local de cada lugar, como a cerveja com o futebol no Brasil, logo que o processo socialização impõem as pessoas, normas e valores que são essenciais para uma harmonia social (PESSÔA, C. A. M, 2012;ACSERALD et al., 2012).

Entretanto existem fatores além do marketing e o fácil acesso ao álcool, que podem levar ao seu consumo, como o incentivo através de amizades, isso se deve principalmente a necessidade de se enquadrar em grupos na sociedade (NEVES, TEIXEIRA, \& FERREIRA, 2015). E por consequência, o seu consumo ocorre cada dia mais cedo (KARLA FERRAZ DOS ANJOS \& SANTOS, 2012).

Não se deve o consumo de álcool somente aos fatores de pré-disposição, mas também existem motivos, muitas vezes específicos, que acarretam esse uso (P et al., n.d.)(FILHO \& Teixeira, 2012). Muitos jovens e adultos utilizam o álcool como porta de entrada para interação com os colegas, ou como solução de problemas pessoais, entre outros (KARLA FERRAZ DOS ANJOS \& SANTOS, 2012).

Verifica-se que os amigos, bem como a interação com os mesmos, compõe um dos principais responsáveis pelo uso de substâncias psicoativas, logo que este meio serve como modelo de comportamento, exercendo pressão social que sujeita e influência as pessoas que convivem em tais grupos. Constata-se também que a importância e a influência dos amigos estão diretamente ligadas não só aos motivos do consumo de álcool, mas também ao primeiro acesso ao mesmo (DIETZ, SANTOS, HILDEBRANDT, \& LEITE, 2011).

O primeiro contato que o jovem tem com o álcool se da principalmente as amizades, os jovens por si só já apresentam características naturais da idade como a curiosidade e a objeção, e quando estes se juntam a um grupo que faz o uso de bebidas alcoólicas acabam sendo instigados a experimentar, logo que querem se sentir semelhantes ao grupo.

Não se deve somente as amizades à culpabilidade do incentivo a experimentar o álcool, o jovem também sofre a influência da mídia em prol do consumo dessas bebidas, bem como a influência dos familiares, este ultimo em minoria ("UNIVERSIDADE FEDERAL DO PARANÁ SONIA APARECIDA NAZÁRIO ESTADUAL VINÍCIUS DE MORAES DE NOVA TEBAS - PR ESTADUAL VINÍCIUS DE MORAES DE NOVA TEBAS - PR,” 2011). 
A motivação é um episódio psicológico de significância para a compreensão do consumo de substâncias psicoativas, essa perspectiva possibilita esclarecer os padrões de consumo de uma substância dada às funções psicológicas específicas desempenhadas por esses comportamentos em cada pessoa que faz o consumo(FILHO \& TEIXEIRA, 2011). Esse artigo traz como objetivo a análise desses motivos pelos quais os jovens e adultos consomem o álcool.

\section{Metodologia}

O presente estudo faz parte do projeto "Identificando o perfil epidemiológico da Obesidade em Vitória da Conquista" idealizado pelo Núcleo de Extensão, Pesquisa e Estudo de Doenças Crônicas - NEPEDC da Faculdade Independente do Nordeste. Trata-se de uma pesquisa do tipo analítica com delineamento transversal e abordagem quantitativa. Tendo como amostra inicial 1117 indivíduos e como amostra final 100 indivíduos do município de Vitória da Conquista - BA.

O procedimento portou-se inicialmente através da aplicação de questionário, instrumento para coleta de dados que continha perguntas pertinente ao estudo, seguido de uma segunda entrevista realizada via ligação telefônica. Esses procedimentos foram realizados com indivíduos aleatórios, com faixa etária entre 20 a 59 anos, considerados jovens adultos.

Os formulários foram subdivididos em seções: Avaliação cognitiva, Informações sócio demográficas, questionário de atividade física, whoqolbref, questionário de hábitos alimentares, questionário de doenças pré-existentes, odontológico, avaliação corporal, depressão, ansiedade, etilismo, anabolizante tabagismo e drogas ilícitas. Foram acrescentadas também outras informações a cerca do consumo de bebidas alcoólicas via ligação telefônica.

Os dados obtidos foram analisados pelo Programa Estatístico SPSS através de análise descritiva. Para a descrição das variáveis contínuas foi empregada a média aritmética, usando o desvio padrão, e para variáveis categóricas utilizou o número absoluto e frequência relativa.

O processo de desenvolvimento desse trabalho foi coordenado de acordo a Resolução 466/12 e submetido à aprovação do Comitê de Ética em Pesquisa da Faculdade Independente do Nordeste - FAINOR com respectivo número 65461317.5.0000.5578. 


\section{Resultados e Discussão}

O presente estudo foi realizado em diferentes pontos na cidade de Vitória da Conquista - BA entre os anos 2016/2017. A população envolvida foram jovens e adultos escolhidos de forma aleatória. Sendo que a amostra final contou com 100 pessoas que optaram participar da pesquisa até o final.

Houve um grau de dificuldade em obter os resultados de todos os participantes do estudo, logo que a segunda etapa do questionário foi realizado através via telefônica e devido a isso, muitos não atenderam as ligações, outros se sentiram inseguros em relatar o uso do álcool, entre outros imprevistos.

Participaram deste estudo 1117 pessoas, dessas, 229 relataram não consumir bebidas alcoólicas, 334 não quiseram relatar o uso ou não da bebida e 554 disseram que faz o uso. Dentre as 554 que relataram fazer o consumo do álcool, apenas 100 se dispôs a responder o questionário.

Tabela 1. Características Sociodemográficas coletadas dos participantes.

\begin{tabular}{lcr}
\hline \multirow{2}{*}{ Variáveis } & $\mathbf{N}$ & Frequência \\
\cline { 2 - 4 } & & \\
\hline Idade & 16 & 16,00 \\
Jovens Adultos & 59 & 59,00 \\
Adultos & 21 & 21,00 \\
Adultos Velhos & 4 & 4,00
\end{tabular}

\section{Gênero}

$\begin{array}{ll}\text { Feminino } & 57\end{array}$

Masculino

\section{Trabalha ou não}

Sim 
Id on Line Revista Multidisciplinar e de Psicoloqia

Id on Line Multidisciplinary and Psycology Journal

Não

23

23,00

Ausente no sistema

1

1,00

\section{Nível de escolaridade}

Fundamental incompleto 5

Fundamental completo

Médio incompleto

Médio Completo 23

Superior incompleto 37

Superior completo

Ausente no sistema

\section{Classe social}

CLASSE C 8

CLASSE D 16

CLASSE E

Ausente no sistema

Total

100,00

Fonte: Dados coletados pela autora em 2017.

Os que se despuseram a responder o questionário são caracterizados por serem jovens e adultos, sendo $16 \%$ Jovens adultos, 59\% Adultos e $21 \%$ Adultos velhos.

Tabela 2. Informações acerca do consumo de álcool pelos jovens e adultos participantes.

\begin{tabular}{lll}
\hline & \multicolumn{3}{c}{ Frequência } \\
\cline { 2 - 3 } Variáveis & $\mathbf{N}$ & $\%$ \\
\hline
\end{tabular}

\section{Motivos do consumo de álcool}

Interação com colegas

Distração de uma 
Id on Line Revista Multidisciplinar e de Psicoloqia

Id on Line Multidisciplinary and Psycology Journal

Aliviar tensão/stress

14

7

14,00

Lazer

4

7,00

Expectativa em algo positivo

Aumento de Confiança com

2

outras pessoas

\section{Lugares mais frequentados para 0 consumo de álcool}

Em casa

Bares

Casas de amigos

Clubes

Primeiro acesso a bebidas alcoólicas

por meio de:

Amigos

Interesse próprio

Parentes

\section{Período que iniciou o consumo}

Adolescência

61

61,00

Fase adulta

39

39,00

Total 100,00

Fonte: Dados coletados pela autora em 2017.

Ao questionar aos pesquisados com que idade iniciou o consumo de álcool, $61 \%$ relataram que foi ainda na adolescência e 39\% já na fase adulta.

Em conformidade com a Organização Mundial de Saúde (WHO), em média 2 bilhões de pessoas ingerem bebidas alcoólicas, sendo vista como uma droga de fácil acesso pertinente do seu valor social associado ao seu consumo, constituindo portanto a droga lícita mais utilizada no mundo (LARANJEIRA, PINSKY, ZALESKI, \& CAETANO, 2007). 
O consumo de bebidas alcoólicas ao decorrer dos anos vem antecedendo cada vez mais na vida dos jovens adultos (FEIJÃO, 2010). Notasse nos dados coletados que a maioria dos entrevistados ingeriu o álcool pela primeira vez ainda na adolescência. Segundo o Instituto Brasileiro de Geografia e Estatística (IBGE) o número de adolescentes do último ano do ensino fundamental que já experimentaram bebidas alcoólicas cresceu em 55\%.

Em relação aos meios que induziram os pesquisados a consumirem bebidas alcoólicas pela primeira vez, $68 \%$ disseram ter experimentado através de amigos, $23 \%$ por interesse próprio/curiosidade e $9 \%$ por meio de parentes.

Um estudo feito em 2010 também em Vitória da Conquista - BA revelou que 32\% dos entrevistados experimentaram bebidas alcoólicas pela primeira vez através de amigos, $31 \%$ por meio de parentes devido ao convívio e $26 \%$ por interesse próprio/curiosidade (KARLA FERRAZ DOS ANJOS \& SANTOS, 2012). É notória a mudança nas estatísticas nesse intervalo de tempo, a influência das amizades vem pesando muito mais nos últimos anos.

Conforme os dados, a principal via de acesso às bebidas alcoólicas se dá por meio de amizades. Um estudo sucedido pela Universidade de Iowa, nos Estados Unidos relata que amigos tem mais influência do que histórico familiar no momento em que o jovem experimenta o álcool pela primeira vez, sendo este o fator de risco prevalecente (SANAGIOTTO, n.d.).

Seguido do interesse próprio, que abrange vários fatores, como a curiosidade em algo novo ou a influência recebida pelo marketing de bebidas alcoólicas que hoje é uma indústria que atua mundialmente associando o consumo da mesma, a diversos prazeres, estilos de vida, entre outros fatores (BASTOS, n.d.).

Ao serem questionados quanto aos motivos que os levam a ingerir bebidas alcoólicas, $57 \%$ disseram ser para interagir com colegas, 16\% para distrair-se de alguma situação/ problema, $14 \%$ para aliviar tensão/stress, $7 \%$ disseram beber por lazer, $4 \%$ em expectativa em algo positivo e $2 \%$ para aumento de confiança em situações com outras pessoas.

Outro estudo publicado pela Revista Portuguesa de Clínica Geral indica que dentre os seus entrevistados 49,7\% faziam o consumo de álcool para acompanhar os amigos. Apesar da distância e das diferenças de crenças e culturas, percebe-se a semelhança em ambos os resultados demostrando os motivos de caráter social como um fator determinante, é visível que além do prazer no consumo do álcool, exista certo esforço por parte dessas pessoas, para satisfazer às expectativas dos grupos (A. REIS et al., 2011). 
Os processos de socialização impõem às pessoas, normas e valores que são essenciais para uma harmonia social. Visto assim, o consumo de bebidas alcoólicas na nossa sociedade está indefinidamente ligado ao próprio enredo que assegura as vivências sociais (Acserald et al., 2012).

Segundo uma pesquisa feita por (BASTOS, n.d.), dentre os contextos sociais de consumo que resultam em maiores estímulo à prática de consumo de bebidas alcóolicas, considera-se a influência dos grupos de amigos, principalmente em momentos festivos.

Nos resultados, dos lugares em que os pesquisados consomem álcool com mais frequência, 34\% disseram ser em casa, $31 \%$ em bares, $29 \%$ em casas de amigos e $6 \%$ em clubes.

Tabela 3. Correlação entre os motivos pelos quais os jovens e adultos fazem o consumo de álcool e quais os lugares mais frequentados.

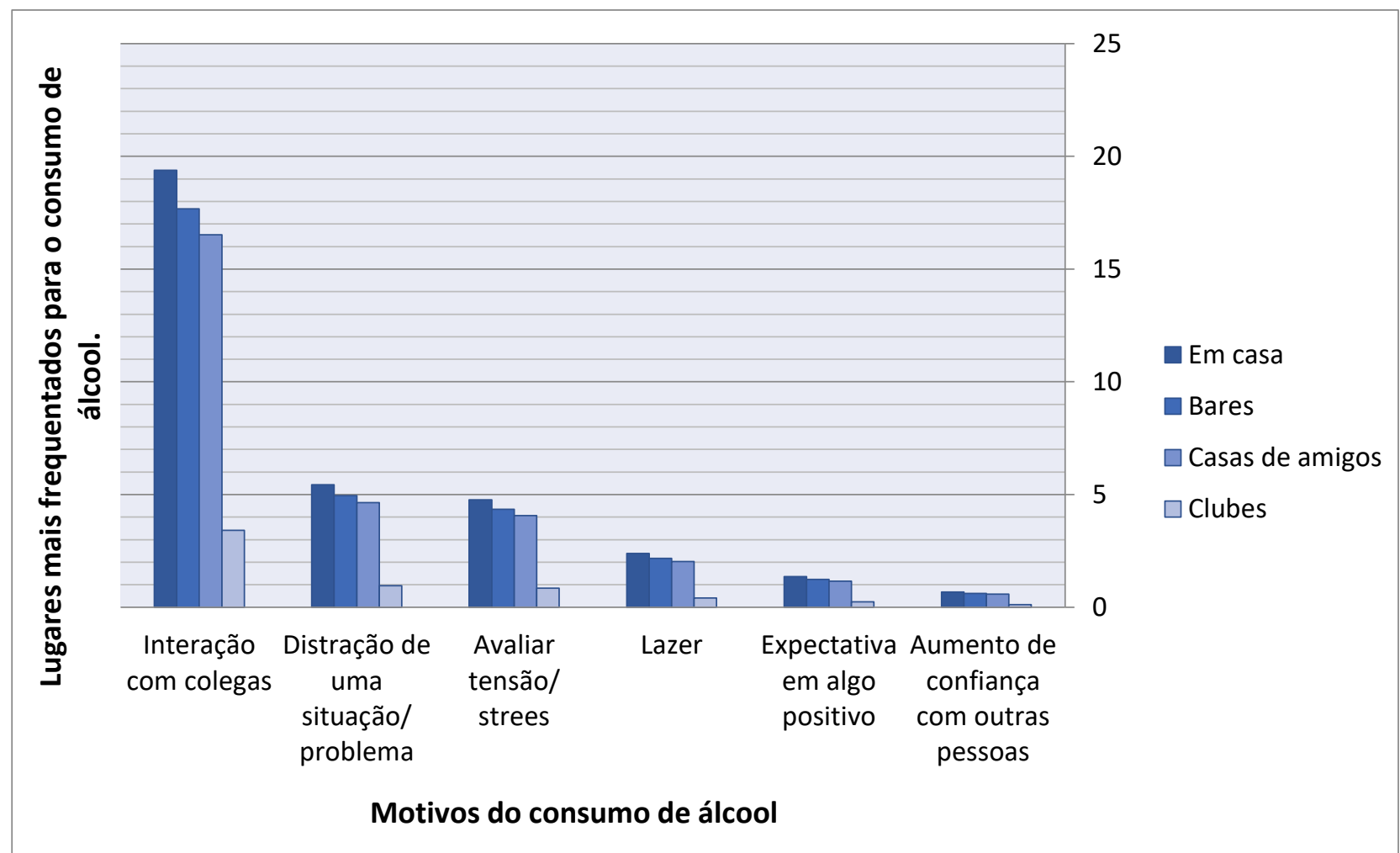

Fonte: Dados coletados pela autora em 2017.

Ao correlacionar os dados obtidos a cerca dos motivos que levam ao consumo de álcool e os lugares de convívio para o consumo com mais frequência, acaba-se sustentando o que já foi argumentado 
acima. Geralmente os principais locais frequentados para socialização entre amigos são representados pelos bares e casa de amigos, que associando estão em maior porcentagem neste estudo.

Também foi relatada no estudo uma grande porcentagem de pessoas que designaram (em casa) como local de mais frequência ao uso do álcool. Porém é importante ressaltar que quando eles se referiam a casa, também estavam em presença de um grupo de amigos. Um dos entrevistados expressou "bebo mais em casa, com meus amigos, eles veem pra cá e a gente bebe por diversão".

O álcool está essencialmente associado às festas, à diversão, ao relaxamento e descontração, totalizando assim um caráter socializador de interação, sendo que a maioria desses locais são coletivos, como bares, casas de amigos, entre outros, esses locais geralmente contêm várias pessoas onde seu utiliza o álcool para aproximação entre as mesmas (ROSA \& NASCIMENTO, 2015).

Tabela 4. Correlação entre o primeiro acesso a bebidas alcoólicas e os motivos do consumo de álcool pelos jovens e adultos.

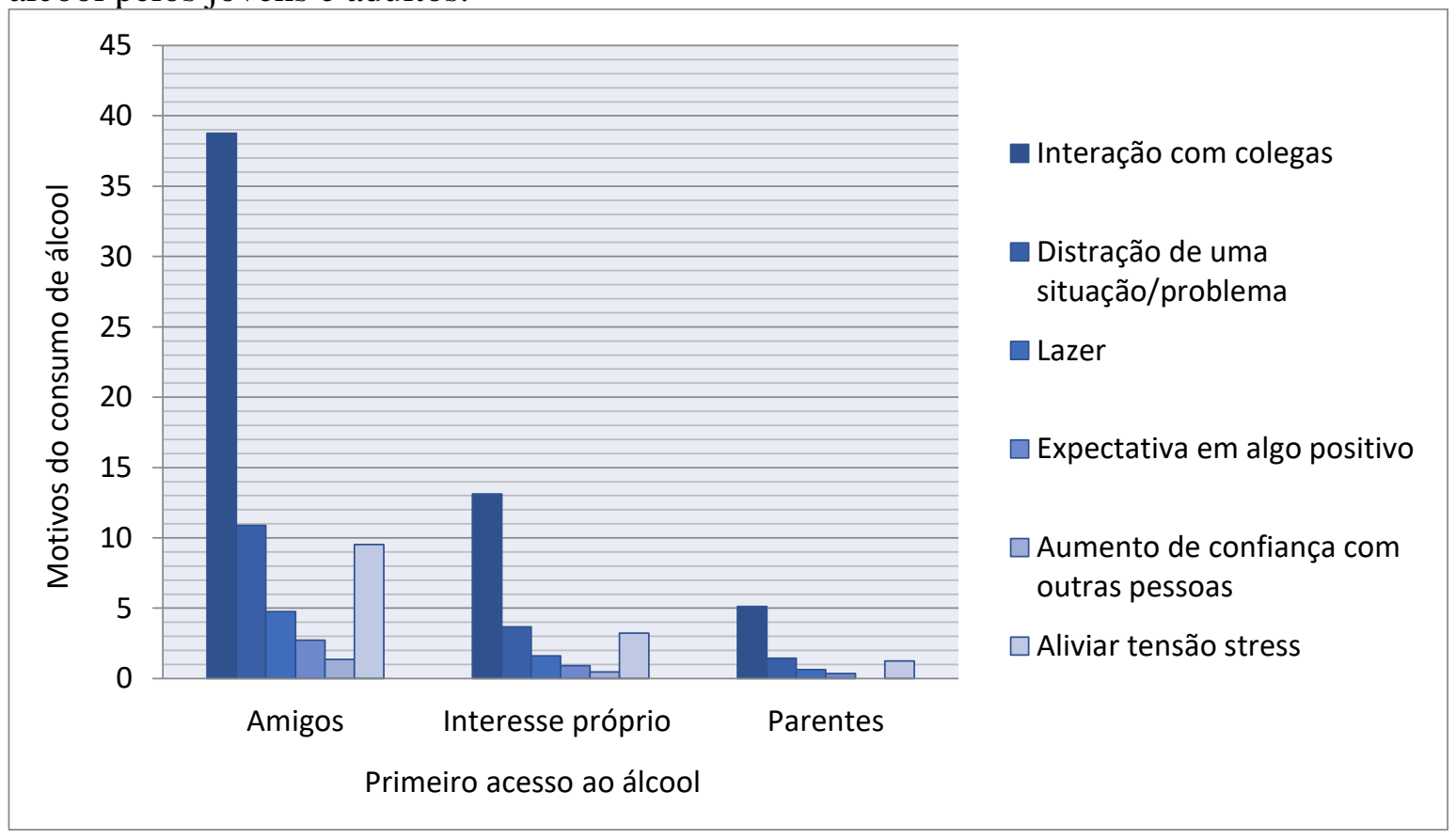

Fonte: Dados coletados pela autora em 2017.

Correlacionando o primeiro acesso a bebidas alcoólicas aos motivos do consumo de álcool pelos jovens e adultos, percebe-se mais uma vez que os amigos sempre estão presentes em maior porcentagem as relações com bebidas com álcool. 
Verifica-se então que a amizade e sua influência torna-se um fator de risco prevalecente tanto no momento em que se tem o primeiro acesso ao álcool, quanto ao uso no cotidiano da vida social (Sanagiotto, n.d.) (A. Reis et al., 2011).

\section{Considerações Finais}

Esta pesquisa foi voltada para análise dos motivos que levam os jovens e adultos a consumirem álcool, e em concordância com a avaliação dos dados e as informações literárias, é possível concluir que este consumo possa ser influenciado por diversos fatores.

Conforme foi descrito ao longo da pesquisa, o marketing e a influência de amigos foram os principais fatores que levaram os participantes a terem o primeiro contato com a bebida. Os resultados obtidos também indicaram que o principal motivo que leva os integrantes da pesquisa a consumir o álcool está atribuído à intenção de interagir com colegas.

Notasse que a sociedade impõe de certo modo, normas e valores que seriam essenciais para uma harmonia social e hoje o uso de bebidas alcoólicas na sociedade está diretamente ligado à própria vivência que assegura uma vida social. Percebe-se então que entre os participantes há uma necessidade do uso da bebida para interagir em grupos sociais, as pessoas se sentem mais a vontade e de certo modo encaixadas naquele meio social.

\section{Referências}

ACSERALD, G., KARAM, M. L., DAVID, H. M. S. L., ALARCON, S., PFEIL, F. M. C., \& ANDRADE, C. S. G. C. De. (2012). Consumo de Bedidas Alcóolicas no Brasil - Estudo com base em fontes secundárias, 162. Retrieved from http://www.flacso.org.br/portal/pdf/area_saude_publica_direitos_humanos/RelatorioConsumo doAlcoolnoBrasilFlacso05082012.pdf

BASTOS, F. V. (n.d.). Motivações para o Consumo de Bebidas Alcoólicas por Jovens: Proposta de uma Tipologia e Recomendações de Marketing Social, 1-15.

DIETZ, G., SANTOS, C. G. dos, HILDEBRANDT, L. M., \& LEITE, M. T. (2011). As Relações Interpessoais e o Consumo de Drogas por Adolescentes. Revista Eletrônica Saúde Mental Álcool E Drogas (SMAD), 7(2), 85-91. Retrieved from http://pepsic.bvsalud.org/scielo.php?pid=S1806-69762011000200006\&script=sci_arttext 
FARIA, R., VENDRAME, A., SILVA, R., \& PINSKY, I. (2011). Propaganda de álcool e associação ao consumo de cerveja por adolescentes. Revista de Saúde Pública, 45(3), 441-447. https://doi.org/10.1590/S0034-89102011005000017

FEIJÃO, F. (2010). Epidemiologia do consumo de álcool entre os adolescentes escolarizados a nível nacional e nas diferentes regiões geográficas. Revista Toxicodependências. Edição IDT, 16, 29-46. Retrieved from http://www.idt.pt/PT/RevistaToxicodependencias/Artigos Ficheiros/2010/1/Text3Vol16_n1E.pdf

FILHO, N. H., \& TEIXEIRA, M. A. P. (2011). Avaliação de motivos para uso de álcool: uma revisão de literatura. PSICO, Porto Alegre, PUCRS, 42(1), 7-15.

FILHO, N. H., \& TEIXEIRA, M. A. P. (2012). Motivos para beber e situações de consumo de bebidas alcoólicas: um estudo exploratório. Mudanças - Psicologia Em Saúde, 20(1-2), 1-6.

KARLA FERRAZ DOS ANJOS, V. C., \& SANTOS, O. da S. A. (2012). Caracterização Do Consumo De Álcool Entre Estudantes Do Ensino Médio. Revista Baiana de Saúde Pública, $36(2), 418-431$.

LARANJEIRA, R., PINSKY, I., ZALESKI, M., \& CAETANO, R. (2007). I Levantamento Nacional Sobre Os Padrões De Consumo De Álcool. Secretaria Nacional de Políticas sobre drogas. Retrieved from http://bvsms.saude.gov.br/bvs/publicacoes/relatorio_padroes_consumo_alcool.pdf

NEVES, K. DO C., TEIXEIRA, M. L. DE O., \& FERREIRA, M. de A. (2015). Factors and motivation for the consumption of alcoholic beverages in adolescence. Escola Anna Nery Revista de Enfermagem, 19(2), 286-291. https://doi.org/10.5935/1414-8145.20150038

P, M. A., ÁGUIDA, M., MOURA, P., LEITE, J., BRAGA, B., SILVA, E., ... LEITE, J. S. (n.d.). PESQUISA Motivação para o consumo de álcool entre adultos jovens em Teresina Motivation for alcohol consumption among young adults in Teresina Motivacion para el consumo de alcohol entre los adultos jóvenes en Teresina presente estudo de praticamente toda, $62-70$.

REIS, A., BARROS, J., FONSECA, C., \& PARREIRA, L. (2011). Prevalência da INGestão de Álcool nos Adolescentes - Estudo PINGA. Revista Portuguesa de Clínica Geral, 27(4), 338346. Retrieved from http://www.scielo.mec.pt/scielo.php?script=sci_arttext\&pid=S0870$71032011000400004 \& \operatorname{lng}=\mathrm{pt} \& \mathrm{nrm}=\mathrm{iso} \& \operatorname{lng}=\mathrm{pt}$

REIS, G. A., GÓIS, H. R. DE, ALVES, M. S., \& PARTATA, A. K. (2014). Alcoolismo e seu Tratamento. Revista Científica Do ITPAC, Araguaína, 7(2), 1-11.

ROSA, L. F. DE A., \& NASCIMENTO, A. R. A. (2015). Arquivos brasileiros de psicologia. Arquivos Brasileiros de Psicologia (Vol. 67). Univ. Retrieved from http://pepsic.bvsalud.org/scielo.php?script=sci_arttext\&pid=S1809- 
$52672015000100002 \& \operatorname{lng}=\mathrm{pt} \& \mathrm{nrm}=\mathrm{iso} \& \mathrm{t} \operatorname{lng}=\mathrm{pt}$

SANAGIOTTO, C. (n.d.). Produções Didático-Pedagógicas.

UNIVERSIDADE FEDERAL DO PARANÁ SONIA APARECIDA NAZÁRIO ESTADUAL VINÍCIUS DE MORAES DE NOVA TEBAS - PR ESTADUAL VINÍCIUS DE MORAES DE NOVA TEBAS - PR. (2011).

VENTUROSO, Edmar. (2015). Breve Estudo Sobre O Consumo De Bebidas Alcoólicas Entre Colaboradores / Funcionários De Uma Cervejaria De Médio Porte Do Centro-Oeste Do Estado De São Paulo. Trabalho de conclusão do curso de Enfermagem da Fundação Educacional do Município de Assis - FEMA, 2015.

\section{Como citar este artigo (Formato ABNT):}

SOARES, Fernanda de Jesus; OLIVEIRA, Daiana C.; OLIVEIRA, Paula R.; LIMA, Tatiane S.; ALVES, Adriana L.R.; SILVA, Matheus L.; DUARTE, Stênio Fernando P. Análise dos Motivos dos Jovens e Adultos consumirem Álcool. Id on Line Revista Multidisciplinar e de Psicologia, Maio de 2017, vol.11, n.35, p.554-566. ISSN: 1981-1179.

Recebido: 29.05.2017

Aceito: 31.05 .2017 